\title{
Linearization problem on structurally finite entire functions
}

\author{
Yûsuke Okuyama* \\ Department of Mathematics, Faculty of Science, \\ Kanazawa University, Kanazawa 920-1192 Japan \\ email; okuyama@kenroku.kanazawa-u.ac.jp \\ Dedicated to the memory of Professor Nobuyuki Suita
}

\begin{abstract}
We show that if a 1-hyperbolic structurally finite entire function of type $(p, q), p \geq 1$, is linearizable at an irrationally indifferent fixed point, then its multiplier satisfies the Brjuno condition. We also prove the generalized Mañé theorem; if an entire function has only finitely many critical points and asymptotic values, then for every such a nonexpanding forward invariant set that is either a Cremer cycle or the boundary of a cycle of Siegel disks, there exists an asymptotic value or a recurrent critical point such that the derived set of its forward orbit contains this invariant set. From it, the concept of $n$-subhyperbolicity naturally arises.
\end{abstract}

\section{Introduction}

A structurally finite entire function is constructed from finitely many quadratic blocks and exponential blocks by Maskit surgeries which connect two functions.

Definition 1.1 (structural finiteness). A structurally finite entire function of type $(p, q)$ is an entire function constructed from $p$ quadratic polynomial blocks and $q$ exponential function blocks. $\mathcal{S F}_{p, q}$ denotes the set of all structurally finite entire functions of type $(p, q)$.

\footnotetext{
${ }^{*}$ Partially supported by the Sumitomo Foundation, and the Ministry of Education,
} Science, Sports and Culture, Grant-in-Aid for Young Scientists (B), 15740085, 2004. 
For the precise definition and details, see [15] or [14. By this definition, we have that a structurally finite entire function has in fact both the topological characterization and the explicit representation, which are used in Section 3 and 4 respectively:

Theorem 1.1 (topological characterization [15]). Every element of $\mathcal{S F}_{p, q}$ has exactly $p$ critical points and $q$ transcendental singularities of its inverse. Conversely, every entire function with exactly $p$ critical points and $q$ transcendental singularities of its inverse belongs to $\mathcal{S} \mathcal{F}_{p, q}$.

In the above and the whole paper, we always count critical points with multiplicities. Hence structurally finite entire functions are of the Speiser class, that is, have finitely many singular values. The classification theorem of Fatou components of this class is known ([6] and [3]). In particular, there are neither wandering nor Baker domains.

Theorem 1.2 (explicit representation [14]). For $(p, q) \neq(0,0), \mathcal{S F}_{p, q}$ agrees with $S F_{p, q}$, where

$$
S F_{p, q}:=\left\{\int_{0}^{z}\left(c_{p} t^{p}+\cdots+c_{0}\right) e^{a_{q} t^{q}+\cdots+a_{1} t} d t+b ; c_{p} a_{q} \neq 0\right\} \quad(q \neq 0),
$$

and $S F_{p, 0}:=$ Poly $_{p+1}=\{$ polynomials of degree $p+1\}$.

From now on, we assume $\lambda=e^{2 \pi i \alpha}(\alpha \in \mathbb{R}-\mathbb{Q})$.

Let us consider an irrationally indifferent cycle of an entire function $f$ of period $n$ with multiplier $\lambda$. It is called a Siegel cycle if every point of this cycle has a neighborhood where the first return map $f^{n}$ is conformally conjugate to $R_{\lambda}(z)=\lambda z$ on the unit disk. Otherwise it is called a Cremer cycle.

The Brjuno condition for $\alpha$ means that

$$
\sum_{n=0}^{\infty} \frac{\log q_{n+1}}{q_{n}}<\infty
$$

where $\left\{p_{n} / q_{n}\right\}$ is the sequence of rational numbers approximating $\alpha$ defined by its continued fraction expansion.

The following shows that an irrationally indifferent cycle is Siegel when $\alpha$ satisfies this Brjuno condition:

Theorem 1.3 (Brjuno [1]). Let $f(z)=\lambda z+\cdots$ be an analytic germ at the origin. If $\alpha$ satisfies the Brjuno condition, then $f$ is (analytically) linearizable, that is, on a neighborhood of the origin, $f$ is conformally conjugate to $R_{\lambda}(z)=\lambda z$ on the unit disk. 
In [16], Yoccoz gave a beautiful alternative proof of this Brjuno theorem and also showed the following theorem in the case of period one. Later we generalized it in the case of arbitrary period:

Theorem 1.4 (Yoccoz [16], Okuyama [11]). If an irrationally indifferent cycle of a quadratic polynomial with multiplier $\lambda$ is a Siegel cycle, then $\alpha$ satisfies the Brjuno condition.

Even in the cubic polynomial case, it is not known whether Theorem 1.4 can be generalized. In the transcendental entire function case, Lukas Geyer showed the following:

Theorem 1.5 (Geyer [5]). If the origin is a Siegel fixed point of $\lambda \int_{0}^{z}(1+$ $t) e^{t} d t$, then $\alpha$ satisfies the Brjuno condition.

Clearly Geyer's example belongs to $\mathcal{S F}_{1,1}$, quadratic polynomials to $\mathcal{S F}_{1,0}$, and both of them naturally satisfy the 1-hyperbolicity defined in Section 2 (see also [11]). In this paper, we shall extend Geyer's result to 1-hyperbolic structurally finite entire functions by more general and synthetic method:

Main Theorem 1. If a 1-hyperbolic structurally finite entire function of type $(p, q), p \geq 1$, has a Siegel fixed point with multiplier $\lambda=e^{2 \pi i \alpha}$, then $\alpha$ satisfies the Brjuno condition.

In the case $p=0$, we just have:

Main Theorem 2. If a 1-hyperbolic structurally finite entire function of type $(0, q), q \geq 1$, has a Siegel fixed point with multiplier $\lambda=e^{2 \pi i \alpha}$, then $E(z)=\lambda \int_{0}^{z} e^{t} d t$ is linearizable at the origin.

We note that $E(z)=\lambda \int_{0}^{z} e^{t} d t \in \mathcal{S F}_{0,1}$. Hence the most fundamental case remains open:

Problem. If the origin is a Siegel fixed point of $E(z)=\lambda \int_{0}^{z} e^{t} d t$, then $\alpha$ satisfies the Brjuno condition?

In Section 2, we define the n-subhyperbolicity in the way similar to that in 11. The generalized Mañé theorem is crucial. In Section 3, we shall explain the linearizability-preserving perturbation of the $n$-hyperbolic entire function, which increases the number of the foliated equivalence classes of acyclic singular values in the Fatou set. The topological characterization of $\mathcal{S} \mathcal{F}_{p, q}$ shows that this perturbation is closed in $\mathcal{S F}_{p, q}$. In Section 4 we shall prove Main Theorems. Using the explicit representation of $\mathcal{S F}_{p, q}$, we can apply the algebraic quadratic perturbation, which was first applied to polynomials by Pérez-Marco [12], to structurally finite entire functions. In Section [5, we shall give a proof of the generalized Mañé theorem, from which we naturally derive the concept of $n$-subhyperbolicity. 
ACKNOWLEDGMENT. The author would like to express his gratitude to Prof. Masahiko Taniguchi and Prof. Toshiyuki Sugawa for valuable discussions and advices, and to Prof. Mitsuhiro Shishikura for commenting on the proof of the corollary of the Mañé theorem.

The author must particularly thanks the referee. The referee read the paper with great care, pointed out many minor mistakes, the important one of which was involved with the generalized Mañé theorem; the author could correct the statement of Theorem [5.1] and refine the proof of Theorem 2.1. The referee's careful comments also helped the author refine the presentation of this article.

\section{$2 n$-subhyperbolicity}

We assume that entire functions are neither constant nor linear. Let $f$ be an entire function and $F(f)$ and $J(f)$ the Fatou and Julia sets of $f$ respectively.

Definition 2.1 (derived set, $\omega$-limit set, and recurrence). The derived set $d(c)$ of $c \in \mathbb{C}$ is defined by the set of all derived (or accumulation) points $z \in \mathbb{C}$ of $\left\{f^{n}(c)\right\}_{n \in \mathbb{N}}$, i.e., $z \in \mathbb{C}$ such that for every neighborhood $U$ of $z$, $(U-\{z\}) \cap\left\{f^{n}(c)\right\}_{n \in \mathbb{N}} \neq \emptyset$. The $\omega$-limit set $\omega(c)$ of $c \in \mathbb{C}$ is defined by the set of all $z \in \mathbb{C}$ such that $\lim _{i \rightarrow \infty} f^{n_{i}}(c)=z$ for some increasing $\left\{n_{i}\right\} \subset \mathbb{N}$. A point $c$ is recurrent if $\omega(c) \ni c$.

Remark. If $c$ is either periodic or preperiodic, then $d(c)=\emptyset$ and $\omega(c)$ equals the cycle where $c$ is eventually mapped. Otherwise $d(c)=\omega(c)$. In particular, if $c$ is a recurrent critical point of $f$ in $J(f), d(c)=\omega(c)$.

Definition 2.2 (correspondence). An asymptotic value or a recurrent critical point $s$ corresponds to an irrationally indifferent cycle $C$ if $d(s) \supset \Gamma$. Here let $\Gamma=\Gamma_{C}$ be, if $C$ is Siegel, the boundary of the cycle of the Siegel disks associated with $C$, otherwise the cycle $C$ itself.

Remark. If $s$ corresponds to an irrationally indifferent cycle, then $d(s) \neq \emptyset$, so $s$ is neither periodic nor preperiodic. It also holds that $s \in J(f)$.

We shall show the following in Section 5 .

Theorem 2.1 (the generalized Mañé theorem). Suppose that $f$ has only finitely many critical points and asymptotic values. Then for every irrationally indifferent cycle $C$, there exists an asymptotic value or a recurrent critical point corresponding to $C$. 
We fix the definition of the transcendental singularities of the inverse of an entire function $f$. For $a \in \mathbb{C}$, let $A:=\{A(r)\}_{r>0}$ be a family of domains in $\mathbb{C}$ such that for $r>0, A(r)$ is a component of $f^{-1}\left(\mathbb{D}_{r}(a)\right)$ and if $0<r_{1}<r_{2}$, then $A\left(r_{1}\right) \subset A\left(r_{2}\right)$. Then the intersection of all the closures of $A(r)$ in $\hat{\mathbb{C}}$ consists of only one point. If this point is the infinity, $A$ is called a transcendental singularity of $f^{-1}$ over $a$. We note that then the $a$ is an asymptotic value of $f$, and that the number of transcendental singularities of $f^{-1}$ is not less than that of asymptotic values.

Definition 2.3 (correspondence). The transcendental singularity $A$ of $f^{-1}$ over the asymptotic value a corresponds to $\Gamma$ if so does $a$.

Convention. For a transcendental singularity $A$ of $f^{-1}$ over $a$, we say that the image of $A$ by $f$ is $a$, and write $f(A)=a$. Moreover, if $a \in J(f)$, we say that $A \in J(f)$.

Now we define the n-subhyperbolicity.

Definition 2.4 ( $n$-subhyperbolicity). Let $f$ be a structurally finite entire function. For a non-negative integer $n, f$ is $n$-subhyperbolic if

(i) there exist exactly $n$ recurrent critical points of $f$ or transcendental singularities of $f^{-1}$ each of which corresponds to some irrationally indifferent cycle of $f$,

(ii) every critical point of $f$ and transcendental singularity of $f^{-1}$ in $J(f)$ other than such ones as in (i) is preperiodic, and

(iii) no orbits of singular values in $F(f)$ accumulate to $J(f)$.

An $n$-subhyperbolic $f$ is $n$-hyperbolic if it has no such (ideal) points as in (ii).

In the case $f$ is a polynomial, this definition agrees with what we have defined in [11]. For several examples of $n$-subhyperbolic polynomials, see [1].

\section{$3 \quad$ Linearizability preserving perturbation}

From now on, we fix $\lambda=e^{2 \pi i \alpha}$, where $\alpha \in \mathbb{R}-\mathbb{Q}$.

For an entire function $f$, a point is said to be acyclic if it is neither periodic nor preperiodic point of $f$. The grand orbit of $x \in \mathbb{C}$ is the set

$$
\left\{y \in \mathbb{C} ; f^{i}(x)=f^{j}(y) \text { for some } i, j \geq 0\right\} .
$$


$x, y \in \mathbb{C}$ are in the foliated equivalence class of $f$ if the closure of their grand orbits agree with each other. Let $N_{A C}(f)$ be the number of the foliated equivalence classes of acyclic singular values of $f$ in $F(f)$.

Proposition 3.1 (linearizability preserving perturbation). Let $f \in$ $\mathcal{S F}_{p, q}$ be $n$-hyperbolic and have a Siegel fixed point with multiplier $\lambda$. Then there exists an $n$-hyperbolic $g \in \mathcal{S F}_{p, q}$ such that

(i) $g$ also has a Siegel fixed point with multiplier $\lambda$, and

(ii) $N_{A C}(g)=p+q-n$.

In the rest of this section, we prove Proposition 3.1. Let $f \in \mathcal{S F}_{p, q}$. We use the following lemmas, the first three of which are about perturbations of critical points, and essentially proved in Section 2 in [11. See also Section 5 in [15], "relaxing the relations between the singularity data".

Lemma 3.1. Let c be a non-periodic critical point in $F(f)$ with multiplicity $m \geq 2$. There exists a Jordan neighborhood $U$ of $c$ in $F(f)$ such that $\bar{U}-\{c\}$ contains no critical point, $f$ maps $U$ onto some Jordan domain properly, and $U \cap \bigcup_{n \geq 1} f^{n}(U)=\emptyset$. And there exist a quasiconformal automorphism $\Phi$ of $\mathbb{C}$ and $g \in \mathcal{S F}_{p, q}$ such that $g$ has exactly $m$ distinct critical points in $\Phi(U)$, which are simple, and $g=\Phi \circ f \circ \Phi^{-1}$ on $\mathbb{C}-\Phi(U)$.

We note that the following Lemma 3.2 is the inverting Carleson and Gamelin operation in [2].

Lemma 3.2. Let c be a periodic critical point in $F(f)$ with multiplicity $m \geq 1$ and of period $p$. There exists a Jordan neighborhood $U$ of $c$ in $F(f)$ such that $\bar{U}$ contains no critical point of $f$ other than $c, f$ maps $U$ onto some Jordan domain properly, and $f^{p}(U) \Subset U$. And there exist a quasiconformal automorphism $\Phi$ of $\mathbb{C}$ and $g \in \mathcal{S F}_{p, q}$ such that $g$ has exactly $m$ distinct critical points in $\Phi(U)$, which are simple, $\Phi(c)$ is not a critical point of $g$, and $g=\Phi \circ f \circ \Phi^{-1}$ on $\mathbb{C}-\Phi(U-\{c\})$.

Lemma 3.3. Let $c$ be a non-periodic and simple critical point in $F(f)$. There exists a Jordan neighborhood $U$ of $c$ in $F(f)$ such that $\bar{U}-\{c\}$ contains no critical point, $f$ maps $U$ onto some Jordan domain properly, and $U \cap \bigcup_{n \geq 1} f^{n}(U)=\emptyset$. For every $y \in f(U)$, there exist a quasiconformal automorphism $\Phi$ of $\mathbb{C}$ and $g \in \mathcal{S F}_{p, q}$ such that $g$ has only one critical point $\Phi(c)$ in $\Phi(U)$, which is simple, $g(\Phi(c))=\Phi(y)$, and $g=\Phi \circ f \circ \Phi^{-1}$ on $\mathbb{C}-\Phi(U)$.

We need to show the following here. In the proof, the classification theorem of Fatou components already stated in Section 1 is implicitly used. 
Lemma 3.4. Let $A=\{A(r)\}_{r>0}$ be a transcendental singularity of $f^{-1}$ over $a \in F(f)$. There exists an $r_{1}>0$ such that $U:=A\left(r_{1}\right)$ is in $F(f)$, disjoint from $\bigcup_{n>0} f^{n}\left(\mathbb{D}_{r_{1}}(a)\right)$, and $\bar{U}$ contains no critical points of $f$. For every $\epsilon \in \mathbb{D}_{r_{1} / 2}$, there exist quasiconformal automorphisms $\Psi$ and $\Phi$ of $\mathbb{C}$ and $g \in \mathcal{S} \mathcal{F}_{p, q}$ such that $\Psi$ is the identity outside $\mathbb{D}_{r_{1}}(a), \Psi(a)=a+\epsilon$, and $g=\Phi \circ \Psi \circ f \circ \Phi^{-1}$ on $\mathbb{C}$. In particular, $g=\Phi \circ f \circ \Phi^{-1}$ on $\mathbb{C}-\Phi(U)$ and $\Phi(a+\epsilon)$ is an asymptotic value of $g$.

Remark. In the above Lemmas, we can assume that the diameter of $U$ is arbitrarily small.

Proof. Since $\bigcup_{n \geq 0} f^{n}\left(\mathbb{D}_{r}(a)\right)$ is uniformly bounded for sufficiently small $r>$ 0 , there exists so small $r_{1}>0$ that $A\left(r_{1}\right) \cap \bigcup_{n \geq 0} f^{n}\left(\mathbb{D}_{r_{1}}(a)\right)=\emptyset$ and $\overline{A\left(r_{1}\right)}$ contains no critical points. Let $\eta:[0,+\infty) \rightarrow[0,1]$ be such a smooth function that identically equals one and zero on $\left[0, r_{1} / 2\right]$ and on $\left[r_{1},+\infty\right)$ respectively. For $\epsilon \in \mathbb{D}_{r_{1} / 2}$, we put $\Psi(w):=w+\epsilon \eta(|w-a|)$. It is easy to see that $\tilde{f}:=\Psi \circ f$ is a quasiregular map on $\mathbb{C}$, equals $f+\epsilon$ and $f$ on $A\left(r_{1} / 2\right)$ and on $\mathbb{C}-A\left(r_{1}\right)$ respectively, and is unbranched on $\overline{A\left(r_{1}\right)}-A\left(r_{1} / 2\right)$. Let $\mu$ be the Beltrami coefficient on $A\left(r_{1}\right)$ of $\tilde{f}$ and we define $\tilde{\mu}$ by the pullback $\left(f^{*}\right)^{n} \mu$ on $f^{-n}\left(A\left(r_{1}\right)\right)(n \in \mathbb{N} \cup\{0\})$ and 0 on $\bigcap_{n \geq 0}\left(\mathbb{C}-f^{-n}\left(A\left(r_{1}\right)\right)\right)$, which is an $\tilde{f}$ invariant Beltrami coefficient on $\mathbb{C}$. Let $\Phi$ be a quasiconformal automorphism of $\mathbb{C}$ whose Beltrami differential equals $\tilde{\mu}$. Then $g=\Phi \circ \tilde{f} \circ \Phi^{-1}$ is an entire function. Finally, since $g$ has the same number of critical points and transcendental singularities as $f$, it follows that $g$ is in $\mathcal{S} \mathcal{F}_{p, q}$ from Theorem 1.1. the topological characterization of structurally finite entire functions.

Proof of Proposition [3.1. Suppose that $f$ is $n$-hyperbolic and have a Siegel fixed point $z_{0}$ with multiplier $\lambda$. By applying the above perturbations to $f$ inductively and in finitely many times, we obtain $g \in \mathcal{S} \mathcal{F}_{p, q}$, which satisfies $N_{A C}(g)=p+q-n$, is $n$-hyperbolic, and is quasiconformally conjugate to $f$ around $z_{0}$ on a neighborhood of $\Phi\left(z_{0}\right)$. Therefore $g$ has a Siegel fixed point $\Phi\left(z_{0}\right)$ with multiplier $\lambda$ (see p. 61-p. 62 in [16]).

\section{Proof of Main Theorems}

Let $f \in \mathcal{S} \mathcal{F}_{p, q}$ have an irrationally indifferent fixed point $z_{0}$ with multiplier $\lambda=e^{2 \pi i \alpha}$, where $\alpha \in \mathbb{R}-\mathbb{Q}$. In the case $q=0$, Main theorem 1] is proved in [11. Therefore we assume $q \geq 1$.

By Theorem 1.2, that is, the explicit representation, and by an affine 
conjugation which maps $z_{0}$ to the origin, we assume that

$$
f(z)=\lambda \int_{0}^{z} P(t) e^{Q(t)} d t
$$

where $P$ is a polynomial of degree $p$ with $P(0)=1$ and $Q$ is that of degree $q$ with $Q(0)=0$. Let $S F_{p, q}(\lambda)$ be the set of all such functions, which is a $(p+q)$-dimensional complex manifold with respect to coefficients of $P$ and $Q$. Furthermore, we say $f_{1} \sim f_{2}\left(f_{1}, f_{2} \in S F_{p, q}(\lambda)\right)$ if $f_{1}(c z) / c=f_{2}$ for some $c \in \mathbb{C}^{*}$.

Suppose that $f$ is 1-hyperbolic and the origin is a Siegel fixed point. By Proposition 3.1, we can also assume that $N_{A C}=p+q-1$, which equals the complex dimension of $S F_{p, q}(\lambda) / \sim$. Since $f$ is 1-hyperbolic, it has no parabolic cycle. Therefore, by the same argument as that in Lemma 4.1 in [11] (see also [10]), the image of the uniformization map (holomorphic injection) from the Teichmüller space of $f$ into $S F_{p, q}(\lambda) / \sim$ becomes a domain in $S F_{p, q}(\lambda) / \sim$. Hence $f$ is quasiconformally stable in $S F_{p, q}(\lambda)$, that is, there exists an open neighborhood of $f$ every element of which is quasiconformally conjugate to $f$.

Proof of Main Theorem 1 . Suppose that $p \geq 1$. Since $f$ is quasiconformally stable in $S F_{p, q}(\lambda)$, there exists a $B>0$ such that for any $|b| \geq B$,

$$
f[b](z):=f(z)+\frac{1}{b} \int_{0}^{z} t e^{Q(t)} d t \in S F_{p, q}(\lambda)
$$

is quasiconformally conjugate to $f$. For any $b \in \mathbb{C}$, we write

$$
F_{b}(z):=\frac{1}{b} f[b](b z)=\lambda z\left(1+\frac{z}{2 \lambda}\right)+\frac{1}{b} h(b z),
$$

where $h$ is an entire function with $h(0)=h^{\prime}(0)=0$.

Proposition 4.1. If $f$ is linearizable at the origin, then

$$
F_{0}(z)=\lambda z\left(1+\frac{z}{2 \lambda}\right)
$$

is also linearizable at the origin.

Proof. We note that for $|b| \geq B, F_{b}$ is linearizable at the origin. As in the case of rational maps (cf. [8] or [9]), we can show that the quasiconformal stability implies the $J$-stability. Hence in fact there exists an $M \geq 0$ such that for $B \leq|b| \leq 2 B$, the Siegel disk of $F_{b}$ at the origin contains $\{|z| \leq M\}$. Then the proposition follows by the same argument as in [12], which is also explained in [11]. For completeness, we write the proof. 
Suppose that $J\left(F_{0}\right)$ intersects $\{|z|<M\}$. Then there exists a $z_{1} \in \mathbb{C}$ with $0<\left|z_{1}\right|<M$ and $n>0$ such that $F_{0}^{n}\left(z_{1}\right)=z_{1}$ since $J\left(F_{0}\right)$ is the closure of the set of all repelling periodic points of $F_{0}$, which is true not only for rational functions but also entire functions. We set:

$$
H(b, z):=\frac{z}{F_{b}^{n}(z)-z}:\{|b|<2 B\} \times\{|z|<M\} \rightarrow \hat{\mathbb{C}}
$$

which depends meromorphically on each variables and is uniformly continuous on $\{|b| \leq 2 B\} \times\{|z| \leq M\}$.

For $B<|b|<2 B$, since $\{|z| \leq M\}$ is contained in the Siegel disk of $F_{b}$ at the origin, $F_{b}$ has no periodic point there. Hence $H(b, z)$ is holomorphic on $\{B<|b|<2 B\} \times\{|z|<M\}$. On the other hand, since $H(b, 0)=1 /\left(\lambda^{n}-1\right)$ is the constant independent of $|b| \leq 2 B$, there exists $0<m<M$ such that $H(b, z)$ is also holomorphic on $\{|b|<2 B\} \times\{|z|<m\}$.

By the Hartogs continuation theorem, $H(b, z)$ is actually holomorphic on $\{|b|<2 B\} \times\{|z|<M\}$. This contradicts the assumption $F_{0}^{n}\left(z_{1}\right)=z_{1}$ and $0<\left|z_{1}\right|<M$.

Hence $F_{0}$ is linearizable at the origin. It follows from this and Theorem 1.4 that $\alpha$ satisfies the Brjuno condition.

Proof of Main Theorem 2. We assume that $f(z)=\lambda \int_{0}^{z} e^{Q(t)} d t, Q(0)=0$. Since $f$ is quasiconformally stable in $S F_{0, q}(\lambda)$, there exists $B>0$ such that for any $|b| \geq B$

$$
f[b](z):=\lambda \int_{0}^{z} e^{Q(t)+t / b} d t \in S F_{0, q}(\lambda)
$$

is quasiconformally conjugate to $f$. For any $b \in \mathbb{C}$, we have

$$
F_{b}(z):=\frac{1}{b} f[b](b z)=\lambda \int_{0}^{z} e^{t} d t+h(b, z),
$$

where $h$ is a holomorphic function on $\mathbb{C} \times \mathbb{C}$ with $h(0, \cdot)=0$.

Proposition 4.2. If $f$ is linearizable at the origin, then

$$
F_{0}(z)=\lambda \int_{0}^{z} e^{t} d t
$$

is also linearizable at the origin.

This can be proved by the same argument as in Proposition 4.1 


\section{Proof of the generalized Mañé theorem}

In this section, we show the generalized Mañé theorem for every entire function with only finitely many critical points and asymptotic values. In [7], he showed it for rational functions (see also [13]). Throughout this section, let $f$ be an entire function with only finitely many critical points and asymptotic values.

Theorem 5.1. Let $M_{f}$ be the set of all asymptotic values and recurrent critical points of $f$, and put $d\left(M_{f}\right):=\bigcup_{s \in M_{f}} d(s)$.

Then there exists an $N \in \mathbb{N}$ such that for every $x \in J(f)-d\left(M_{f}\right)$ which is not a parabolic periodic point and for every $\epsilon>0$, there exists a connected neighborhood $U$ of $x$ such that for every $n \geq 0$ and every connected component $V^{\prime}$ of $f^{-n}(U)$ bounded in $\mathbb{C}$,

(i) the spherical diameter of $V^{\prime}$ is less than $\epsilon$ and $\operatorname{deg}\left(f^{n}: V^{\prime} \rightarrow U\right) \leq N$.

(ii) For every $\epsilon_{1}>0$, there exists an $n_{0} \in \mathbb{N}$ such that for every $n>n_{0}$, the spherical diameter of $V^{\prime}$ is less than $\epsilon_{1}$.

Theorem 5.1 can be shown by completely the same way as Theorem 1.1 in [13. The only difference is that we should exclude not only the $\omega$-limit set of a recurrent critical point but also the derived set of an asymptotic value.

Theorem 5.2, which is not needed to show Theorem 2.1. follows from Theorem 5.1 and is also proved by almost the same argument as Theorem 1.2 in 13 although some extra argument for excluding unbounded iterated preimages of the $U$ in Theorem 5.2 is needed.

Theorem 5.2. Let $\Lambda \subset J(f)$ be compact and forward invariant, i.e., $f(\Lambda) \subset$ $\Lambda$, and contain none of critical points, parabolic periodic points and asymptotic values. If $\Lambda \cap d\left(M_{f}\right)=\emptyset$, then it is expanding; i.e., there exists an $n_{1}>0$ such that for every $n \geq n_{1}, \min _{z \in \Lambda}\left|\left(f^{n}\right)^{\prime}(z)\right|>1$.

Proof. Assume that $\Lambda$ is not expanding. Then there exist $n_{k} \rightarrow \infty, \Lambda \ni z_{k}$, and $x \in \Lambda$ such that $\left|\left(f^{n_{k}}\right)^{\prime}\left(z_{k}\right)\right| \leq 1$ and $\lim _{k \rightarrow \infty} f^{n_{k}}\left(z_{k}\right)=x$. If $x \in$ $\mathbb{C}-d\left(M_{f}\right)$, then $x$ satisfies the condition of Theorem [5.1. For every $\epsilon>0$ such that the spherical $\epsilon$-neighborhood $\Lambda_{\epsilon}$ of $\Lambda$ contains no critical point, let $U$ be a neighborhood of $x$ associated to $\epsilon$ given by Theorem [5.1. Since $\lim _{k \rightarrow \infty} f^{n_{k}}\left(z_{k}\right)=x$, there exists $K(U)>0$ such that $f^{n_{k}}\left(z_{k}\right) \in U$ for every $k>K(U)$.

We show that there exists a $k>K(U)$ such that the component $V_{k}$ of $f^{-n_{k}}(U)$ containing $z_{k}$ is unbounded: otherwise, $f^{n_{k}}: V_{k} \rightarrow U$ is bijective for every $k>K(U)$ since for $0 \leq j \leq n_{k}, f^{j}\left(V_{k}\right)$ is a bounded connected 
component of $f^{-n_{k}+j}(U)$ intersecting $\Lambda$ so is contained in $\Lambda_{\epsilon}$ by (i) in Theorem 5.1. Furthermore, every limit function $\phi$ on $U$ of the single-valued branches $\left\{f^{-n_{k}}: U \rightarrow V_{k}\right\}_{k>K(U)}$ is a constant one by (ii) in Theorem 5.1. This contradicts that $\left|\phi^{\prime}(x)\right|=\lim _{k \rightarrow \infty}\left|\left(f^{n_{k}}\right)^{\prime}\left(z_{k}\right)\right|^{-1} \geq 1$.

For an unbounded $V_{k}$, there exists a $1 \leq j \leq n_{k}$ such that $f^{j}\left(V_{k}\right)$ is bounded but $f^{j-1}\left(V_{k}\right)$ is unbounded. Then $f^{j}\left(V_{k}\right)$ contains an asymptotic value, and is contained in $\Lambda_{\epsilon}$. Consequently, if $\epsilon>0$ is small enough, $\Lambda_{\epsilon}$ contains some asymptotic value. Since $f$ has only finitely many asymptotic values, it implies $\Lambda$ itself contains some asymptotic value. This is a contradiction.

Now we shall prove Theorem 2.1] the generalized Mañé theorem, stated in Section 2, For Cremer cycles, we need some careful argument for finding bounded iterated preimages of the $U$ in Theorem 5.2

Proof. As in Section 2, let $C$ be an irrationally indifferent cycle of period $p$, and $\Gamma=\Gamma_{C}$ the boundary of the cycle of the Siegel disks associated with $C$ if $C$ is Siegel, and the cycle $C$ itself otherwise.

(Cremer Case) Assume that $\Gamma$ is a Cremer cycle. Theorem 5.2 implies that if $\Gamma$ contains no asymptotic value, then $\Gamma \subset d\left(M_{f}\right)$. We shall show that the latter always occurs.

Assume that $\Gamma \cap d\left(M_{f}\right)=\emptyset$. Let $W$ be the bounded and open spherical $\epsilon$-neighborhood of $\Gamma$. Since $\Gamma$ is a finite set and $f$ has only finitely many critical or asymptotic values, there exists an $\epsilon>0$ such that each connected component of $W-\Gamma$ is a spherical once-punctured disk whose puncture is in $\Gamma$ and $W-\Gamma$ contains none of critical or asymptotic values. Then $f: f^{-1}(W-\Gamma) \rightarrow W-\Gamma$ is unbranched. Hence $f$ maps each connected component $Y$ of $f^{-1}(W-\Gamma)$ onto a connected component $X$ of $W-\Gamma$ as a covering map, which is known to be isomorphic to that onto $\mathbb{D}^{*}$ given by either the logarithm or the $n$th root for some $n \in \mathbb{N}$ (cf. 4], Theorem 5.10). $f: Y \rightarrow X$ is a logarithmic covering if and only if $Y$ is simply connected. We note that $f^{-1}(\Gamma)$ is the set of all punctures of $f^{-1}(W-\Gamma)$, and $\Gamma\left(\subset f^{-1}(\Gamma)\right)$ contains no critical point. Hence if $Y$ has its puncture in $\Gamma$, then $f: Y \rightarrow X$ is conformal, and $f$ gives a homeomorphism between the closures of $X$ and $Y$. In particular, since $X$ is bounded, so is $Y$.

By filling the punctures, we conclude that every connected component of $f^{-1}(W)$ that intersects $\Gamma$ is bounded and $f$ maps it onto $W$ conformally. Let $W_{1}$ be the union of all these (only finitely many) connected components of $f^{-1}(W)$ that intersect $\Gamma$.

For every $x \in \Gamma$, which satisfies the assumption of Theorem [5.1 there exists an open neighborhood $U_{x}$ of $\Gamma$ associated to $\epsilon$ given by Theorem 5.1 . 
Let $U:=\bigcup_{x \in \Gamma} U_{x}$. For $k \geq 0$, let $V_{k}$ be the union of such components of $f^{-k}(U)$ that intersect $\Gamma$. By induction, we show $V_{k} \subset W$ : for $k=0$, it is trivial. Assume that it is true for $k$. Since $V_{k+1}$ is the union of such components of $f^{-1}\left(V_{k}\right)$ that intersect $\Gamma, V_{k+1} \subset W_{1}$, which is bounded, so $V_{k+1} \subset W$ by (i) in Theorem 5.1 .

Since $W$ contains no critical point, $f^{k}$ maps $V_{k}$ onto $U$ conformally. By (ii) in Theorem 5.1 every limit function $\phi_{x}$ on $U_{x}$ of the single-valued branches $\left\{f^{-k p}: U_{x} \rightarrow V_{k p}\right\}_{k \geq 0}$ is constant. This contradicts that $\left|\phi_{x}^{\prime}(x)\right|=$ $\lim _{k \rightarrow \infty}\left|\left(f^{k p}\right)^{\prime}(x)\right|^{-1}=1$.

Hence $\Gamma \subset d\left(M_{f}\right)$, which concludes that $\Gamma \subset d(s)$ for some $s \in M_{f}$.

(Siegel case) Next, assume that $\Gamma$ is the boundary of a cycle of the Siegel disks.

Let $A(f)$ be the set of all asymptotic values. Assume that there exists $x \in\left(\Gamma-\bigcup_{n \geq 0} f^{n}(A(f))\right)-d\left(M_{f}\right)$. Then if a neighborhood of $x$ is small enough, it does not intersect $\bigcup_{n \geq 0} f^{n}(A(f))$. Since $x$ satisfies the assumption of Theorem 5.1, there exists an open neighborhood $U \subset \mathbb{C}-\bigcup_{n \geq 0} f^{n}(A(f))$ of $x$ associated to some $\epsilon>0$ given by Theorem 5.1. Since $U$ intersects a Siegel disk, there exist $\epsilon_{1}>0$ and $n_{k} \rightarrow \infty$ such that for every $k \in \mathbb{N}$, the spherical diameter of the connected component $V_{k}$ of $f^{-n_{k}}(U)$ intersecting $\Gamma$ is more than $\epsilon_{1}$. On the other hand, since $U \subset \mathbb{C}-\bigcup_{n>0} f^{n}(A(f)), V_{k}$ is bounded for every $k \in \mathbb{N}$. Hence by (ii) in Theorem [5.1 there exists $n_{0} \in \mathbb{N}$ such that the spherical diameter of $V_{k}$ is less than $\epsilon_{1}$ for every $k>n_{0}$. This is a contradiction.

Hence $\Gamma-\bigcup_{n \geq 0} f^{n}(A(f)) \subset d\left(M_{f}\right)$. Since the left hand side is dense in $\Gamma$ and $d\left(M_{f}\right)$ is closed, it follows that $\Gamma \subset d\left(M_{f}\right)$.

The proof of that $\Gamma \subset d(s)$ for some $s \in M_{f}$ is almost the same as the proof of the original Mañé theorem, so we give its outline and omit the details.

Let $D$ be one of the Siegel disks, $\hat{\Gamma}$ the boundary of $D$ considered in $\hat{\mathbb{C}}$, and $\mu$ the harmonic measure on $\hat{\Gamma}$ with respect to the Siegel periodic point $z_{0} \in D$. The support of $\mu$ equals $\hat{\Gamma}$. In particular, $\mu(\hat{\Gamma}-\{\infty\})>0$.

The dynamical system $\left(\hat{\Gamma}-\{\infty\}, f^{p}|(\hat{\Gamma}-\{\infty\}), \mu|(\hat{\Gamma}-\{\infty\})\right)$ has such an ergodic property that if $f^{p}(B) \subset B$ for a Borel subset $B \subset \hat{\Gamma}-\{\infty\}$, then $\mu(B)$ equals 0 or 1 . In particular, for every $s \in \hat{\mathbb{C}}, \mu((\hat{\Gamma}-\{\infty\}) \cap d(s))$ equals 0 or 1 .

Consequently, since $\hat{\Gamma}-\{\infty\} \subset d\left(M_{f}\right)=\bigcup_{s \in M_{f}} d(s)$, there exists an $s \in M_{f}$ such that $\mu((\hat{\Gamma}-\{\infty\}) \cap d(s))=1$. Hence $\hat{\Gamma} \cap d(s)$ is a $\mu$-full measure closed set in $\hat{\mathbb{C}}$, so contains supp $\mu=\hat{\Gamma} \supset \partial D$, which is the boundary of $D$ in $\mathbb{C}$. Hence $d(s) \supset \partial D$ so $d(s) \supset \overline{\bigcup_{n=1}^{p} f^{n}(\partial D)}=\Gamma$. 


\section{References}

[1] Bruuno, A. D. Analytical form of differential equations, Trans. Moscow Math. Soc., 25 (1971), 199-239.

[2] Carleson, L. and Gamelin, T. W. Complex Dynamics, SpringerVerlag (1993).

[3] Eremenko, A. E. and Lyubich, M. Y. Dynamical properties of some classes of entire functions, Ann. Inst. Fourier, 42 (1992), 989-1020.

[4] Forster, O. Lectures on Riemann Surfaces, Springer-Verlag (1981).

[5] Geyer, L. Siegel discs, Herman rings and the Arnold family, Trans. Amer. Math. Soc., 353, 9 (2001), 3661-3683 (electronic).

[6] Goldberg, L. R. and Keen, L. A finiteness theorem for a dynamical class of entire functions, Ergodic Theory Dynam. Systems, 6, 2 (1986), 183-192.

[7] Mañé, R. On the theorem of Fatou, Bol. Soc. Bras. Mat., 24 (1993), $1-11$.

[8] Mañé, R., Sad, P. and Sullivan, D. On the dynamics of rational maps, Ann. Sc. E.N.S., 4ème série, 16 (1983), 193-217.

[9] McMullen, C. Complex Dynamics and Renormalization, Ann. of Math. Studies, Princeton (1994).

[10] McMullen, C. and Sullivan, D. Quasiconformal Homeomorphisms and Dynamics III : The Teichmüller space of a holomorphic dynamical system, Adv. Math., 135 (1998), 351-395.

[11] Okuyama, Y. Non-linearizability of $n$-subhyperbolic polynomials at irrationally indifferent fixed points, J. Math. Soc. Japan, 53, 4 (2001), $847-874$.

[12] PÉrez-Marco, R. Sur les dynamique holomorphes non linéarisables et une conjecture de V. I. Arnold, Ann. Scient. Éc Norm Sup., $4^{e}$ série, 26 (1993), 565-644.

[13] Shishikura, M. and TAN, L. An alternative proof of Mañé's theorem on non-expanding Julia sets, The Mandelbrot set, theme and variations, Vol. 274, Cambridge Univ. Press, Cambridge (2000), 265-279. 
[14] Taniguchi, M. Explicit representation of structurally finite entire functions, Proc. Japan Acad. Ser. A Math. Sci., 77, 4 (2001), 68-70.

[15] Taniguchi, M. Synthetic deformation space of an entire function, Value distribution theory and complex dynamics (Hong Kong, 2000), Vol. 303 of Contemp. Math., Amer. Math. Soc., Providence, RI (2002), $107-136$.

[16] Yoccoz, J.-C. Théorème de Siegel, nombres de Bruno et polynômes quadratiques, Astérisque, 231 (1995), 3-88, Petits diviseurs en dimension 1 . 